\title{
MSW Effects on High-Energy Solar Neutrinos from Relic Annihilation
}

\author{
John ELLIS, Ricardo A. FLORES* and Samina S. MASOOD ${ }^{\dagger}$ \\ Theory Division \\ CERN \\ 1211 Geneva 23, Switzerland
}

\begin{abstract}
We demonstrate that Mikheyev-Smirnov-Wolfenstein (MSW) matter oscillations can alter significantly the fluxes of high-energy $(\mathrm{GeV})$ solar neutrinos, produced for example by the annihilation of cold dark matter particles in the solar core. We use as illustrations models of neutrino masses and mixings motivated by the low-energy solar neutrino data, and show that the flux of $\nu_{e}$ may be dramatically enhanced and the flux of $\nu_{\mu, \tau}$ correspondingly suppressed, or vice versa. This possible high-energy MSW effect is therefore a source of ambiguity in the interpretation of experimental searches for dark matter annihilations inside the Sun, as we discuss explicitly for sneutrinos, and could eventually be used to discriminate between different solutions to the low-energy solar neutrino problem.
\end{abstract}

CERN-TH 6616/92

UMSL-TH-4/92

August 1992

* Permanent Address: Department of Physics and Astronomy, University of Missouri, Saint Louis, MO 63121 USA.

$\dagger$ Permanent Address: Department of Physics, Quaid-i-Azam University, Islamabad, Pakistan. 


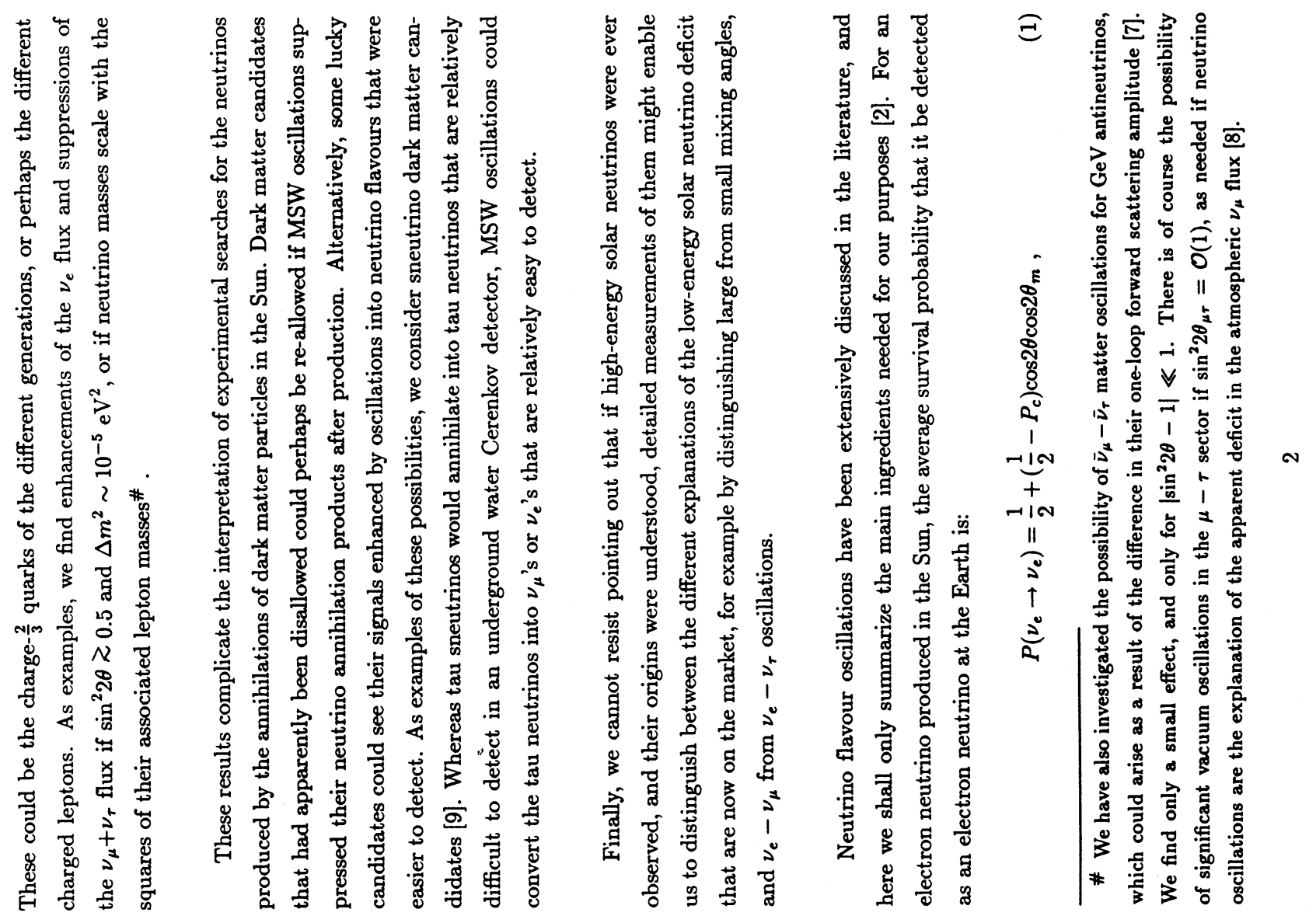

In

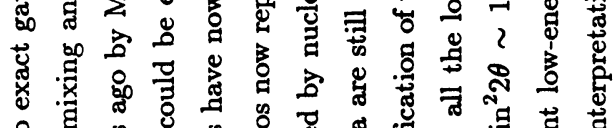
(1) III In

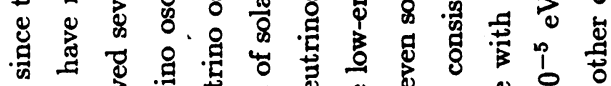

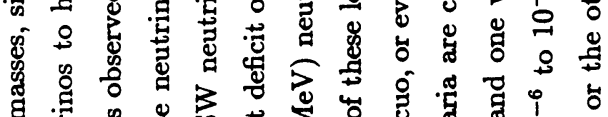

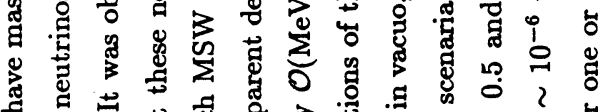

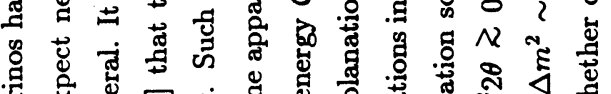
I = In 竞量高 t III IIIIIIIIII

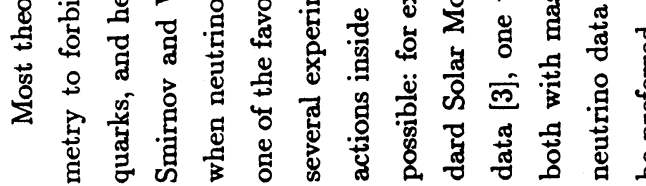



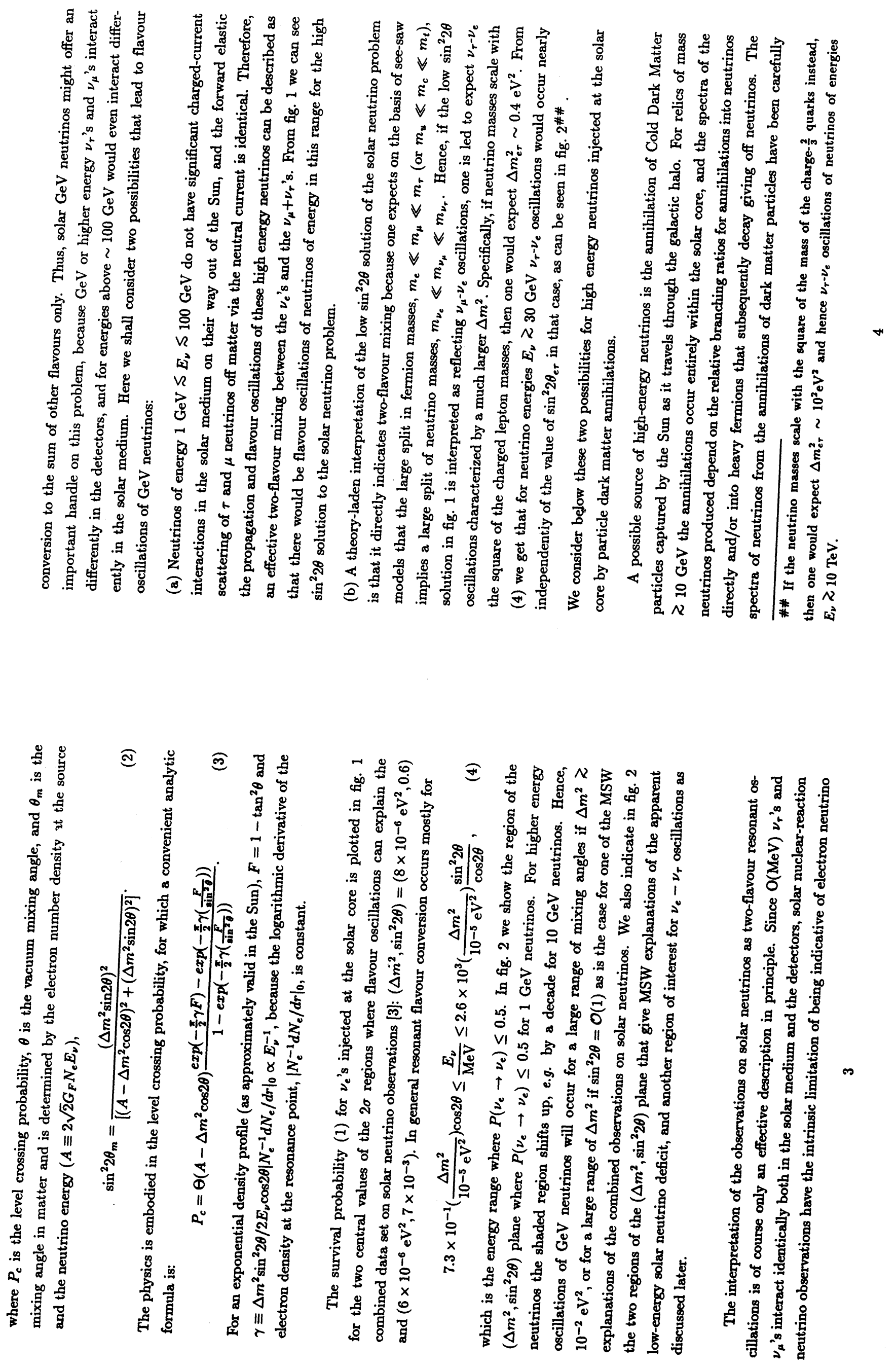


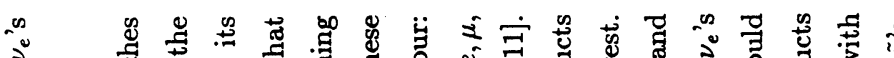

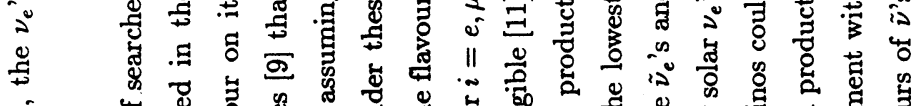

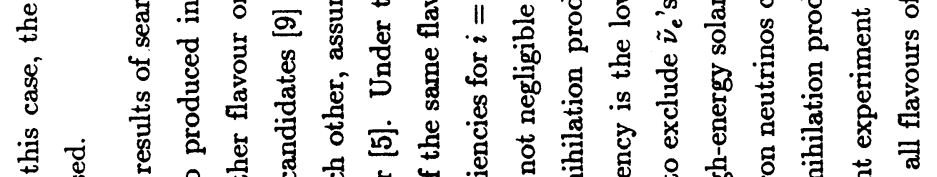

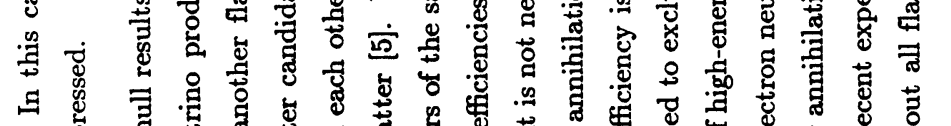

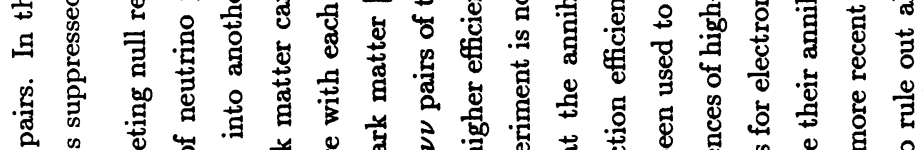

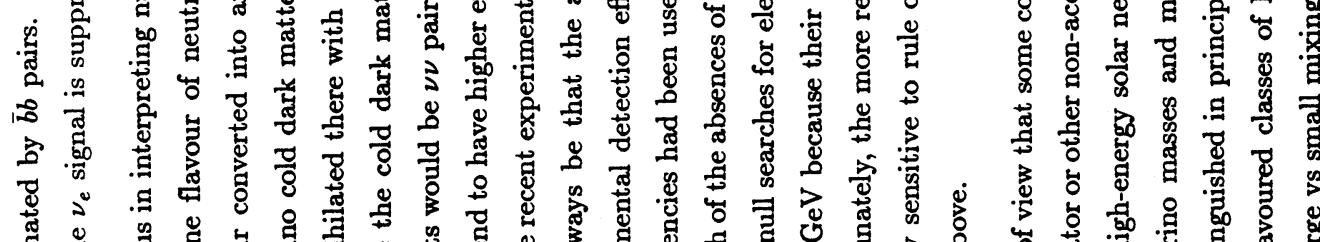

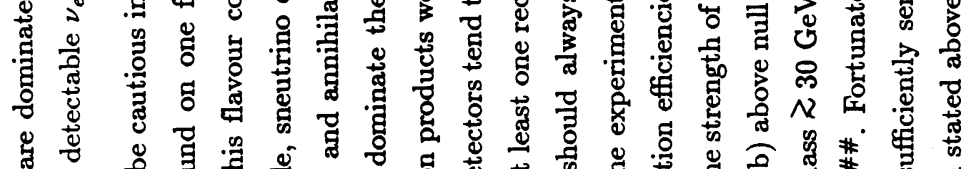

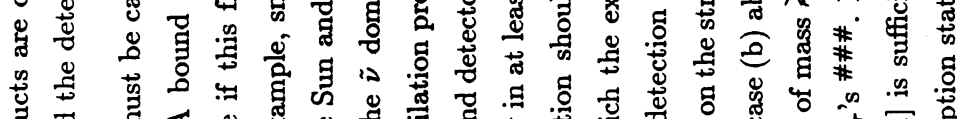

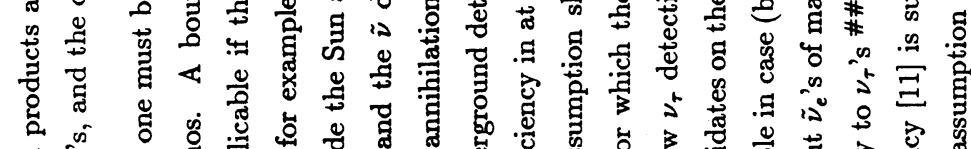

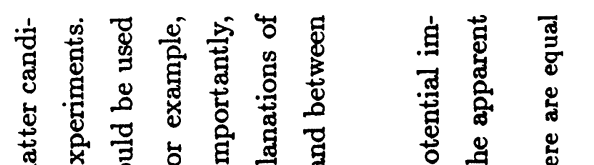

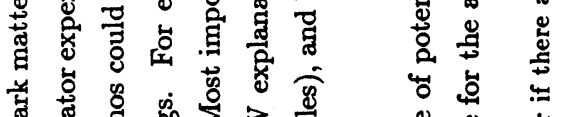

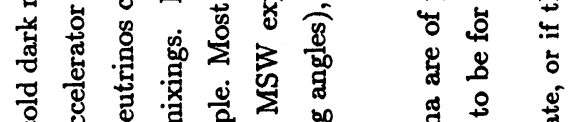

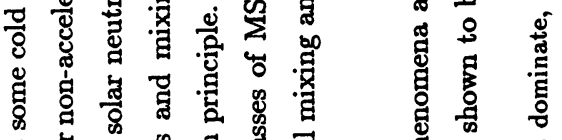

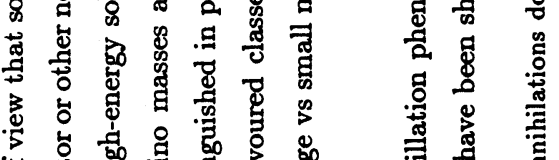

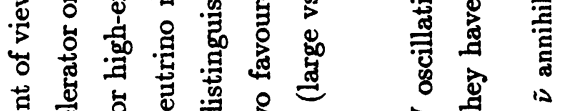

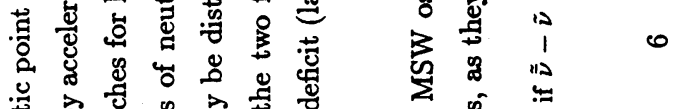

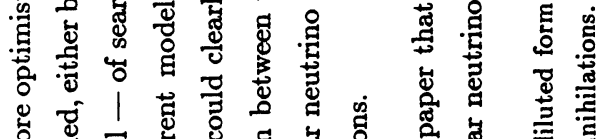

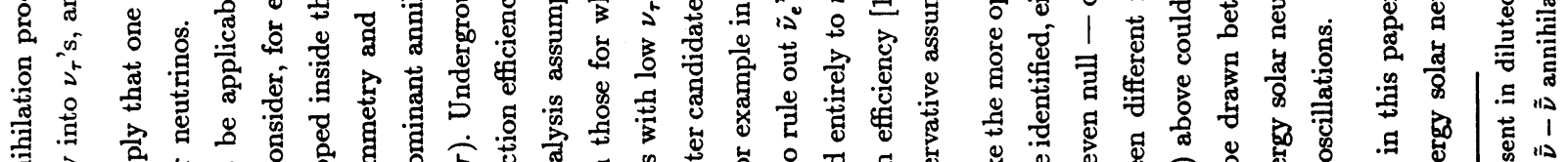

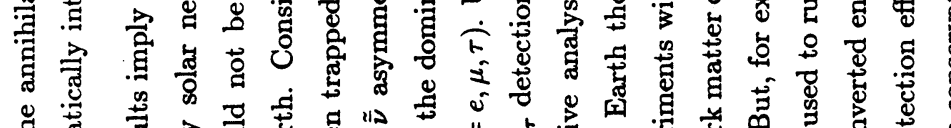

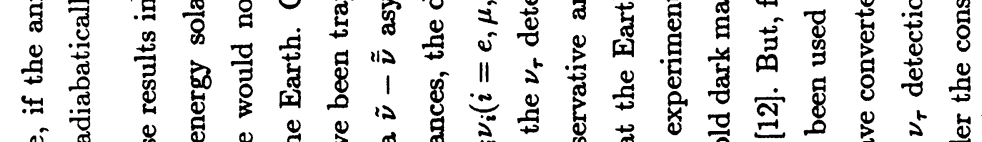

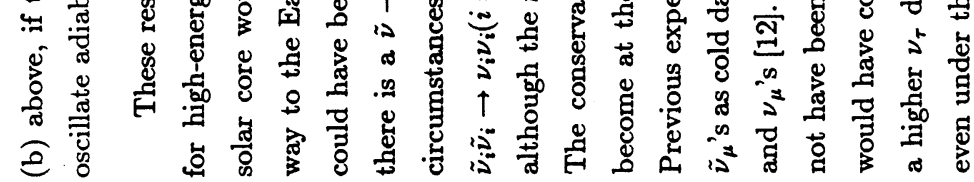

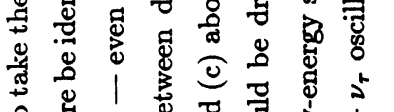

H.

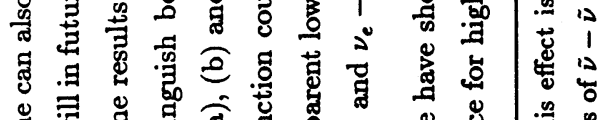

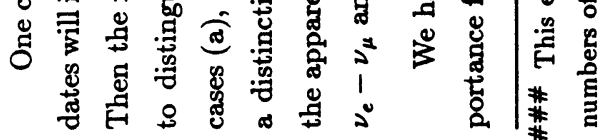

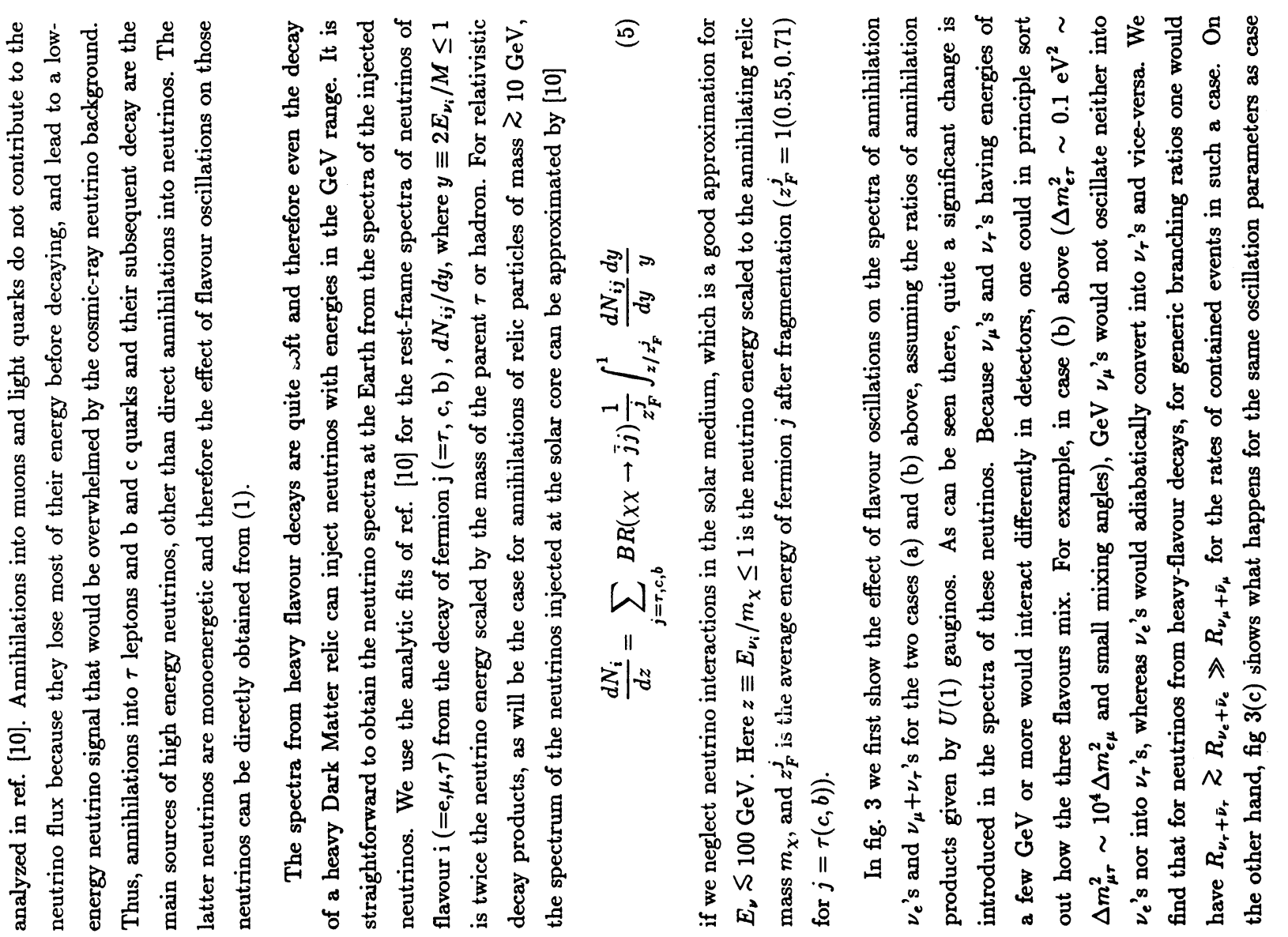



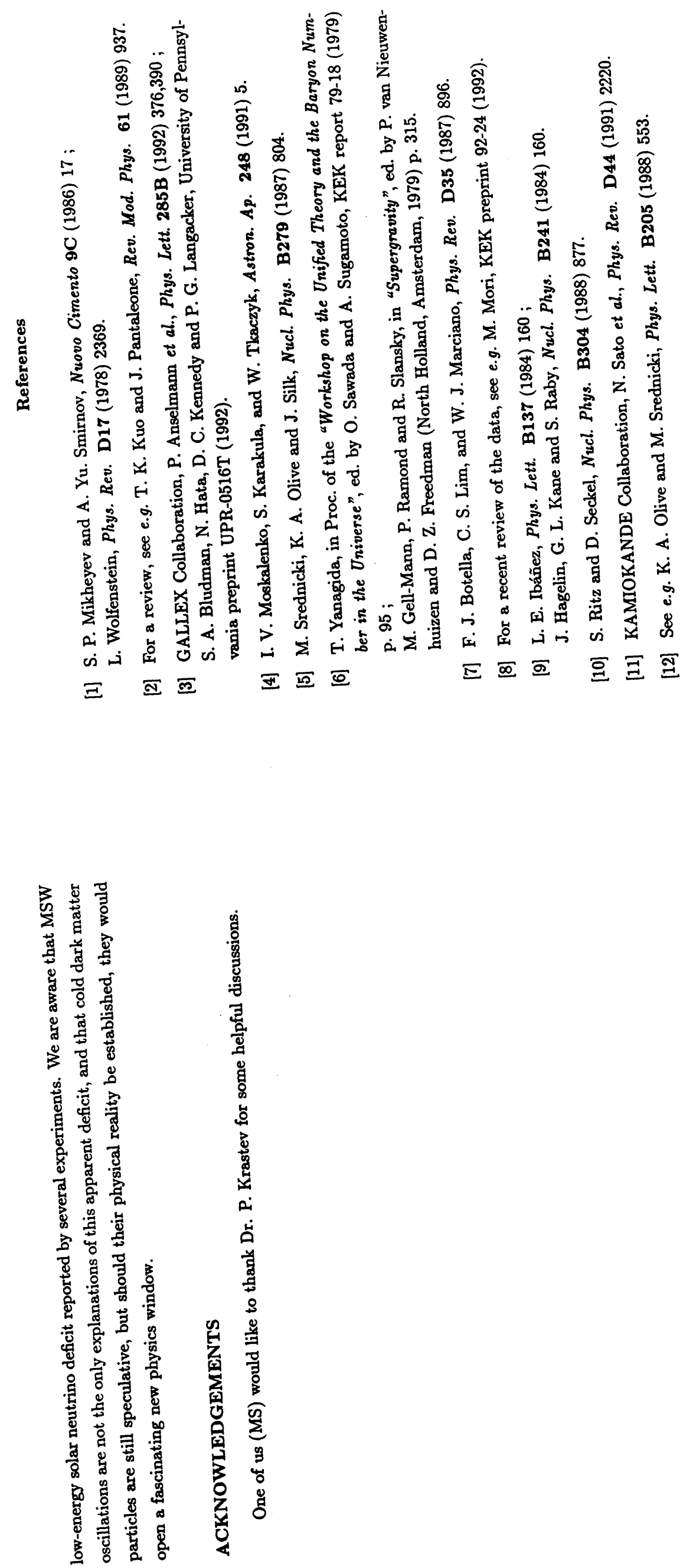

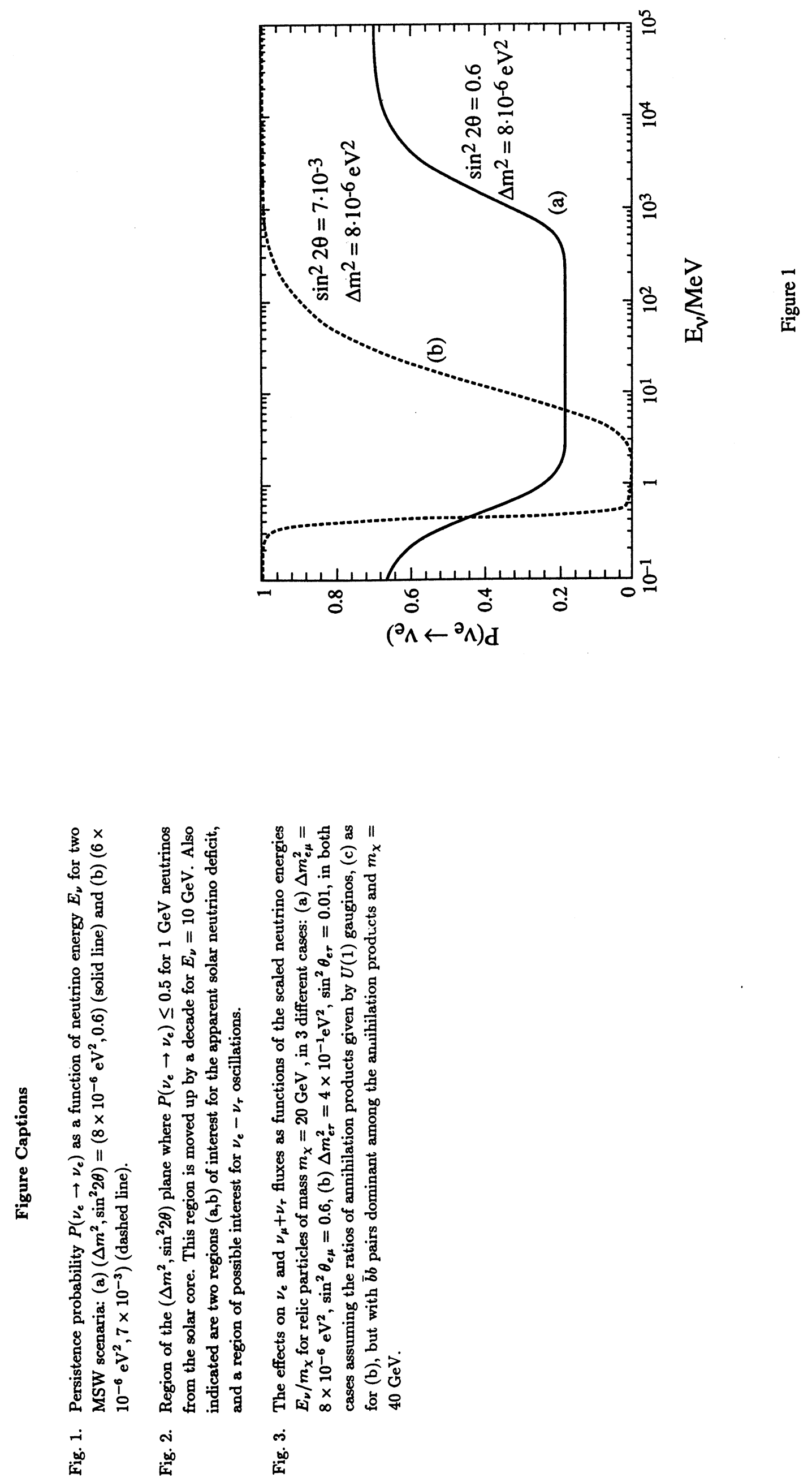

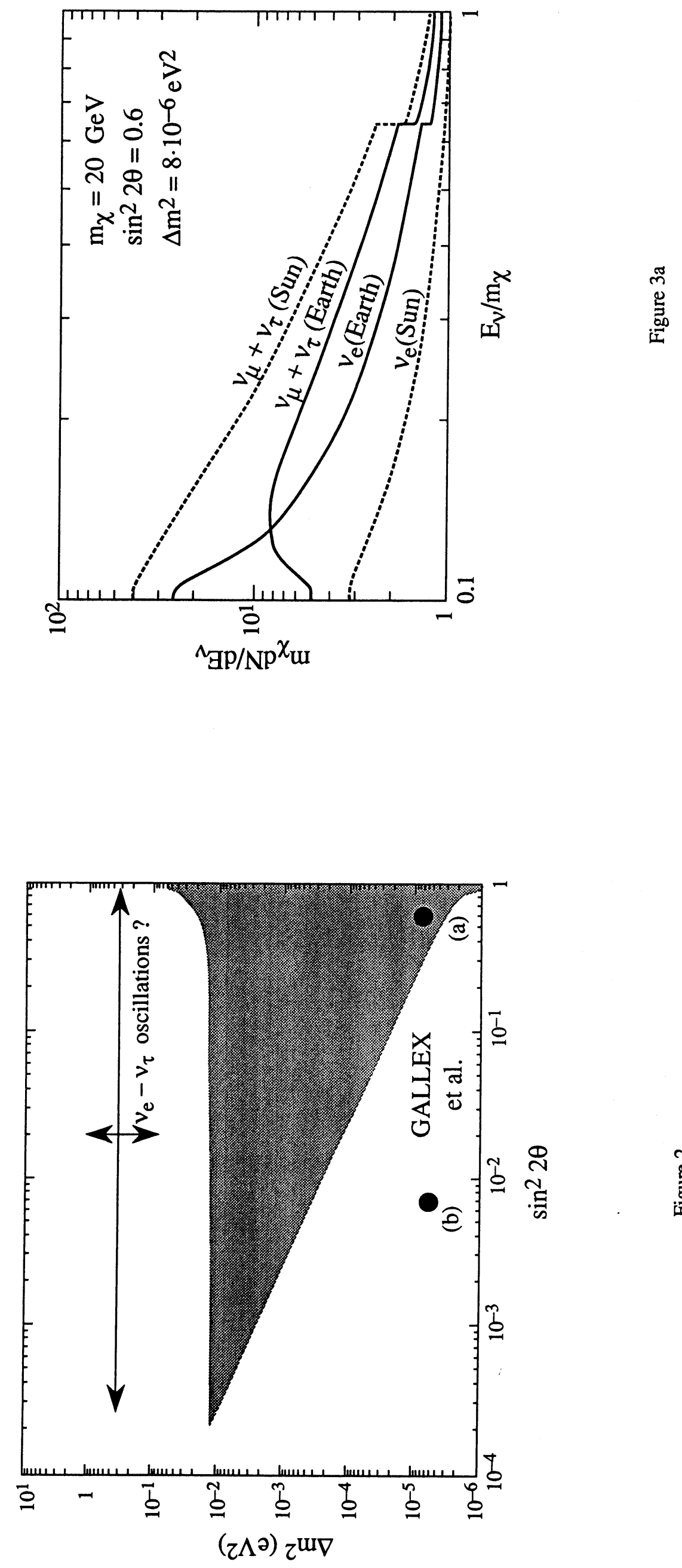

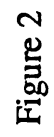




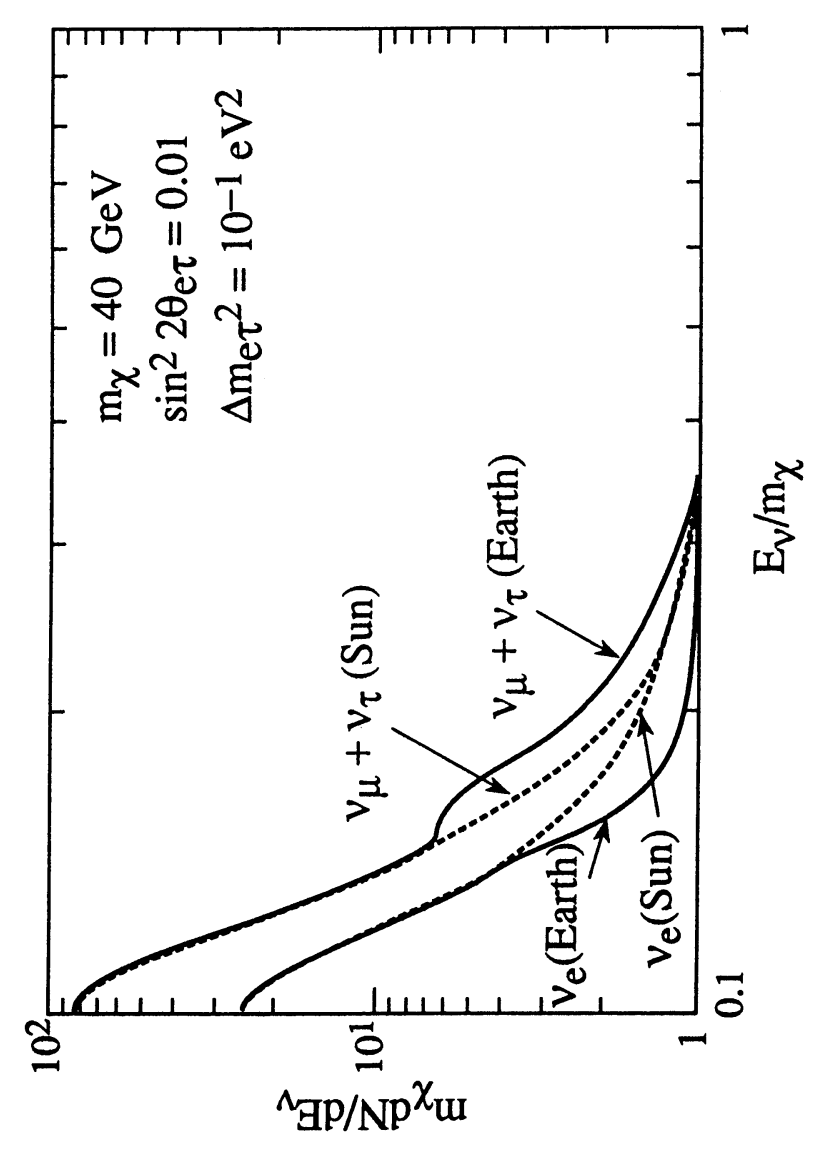

弚

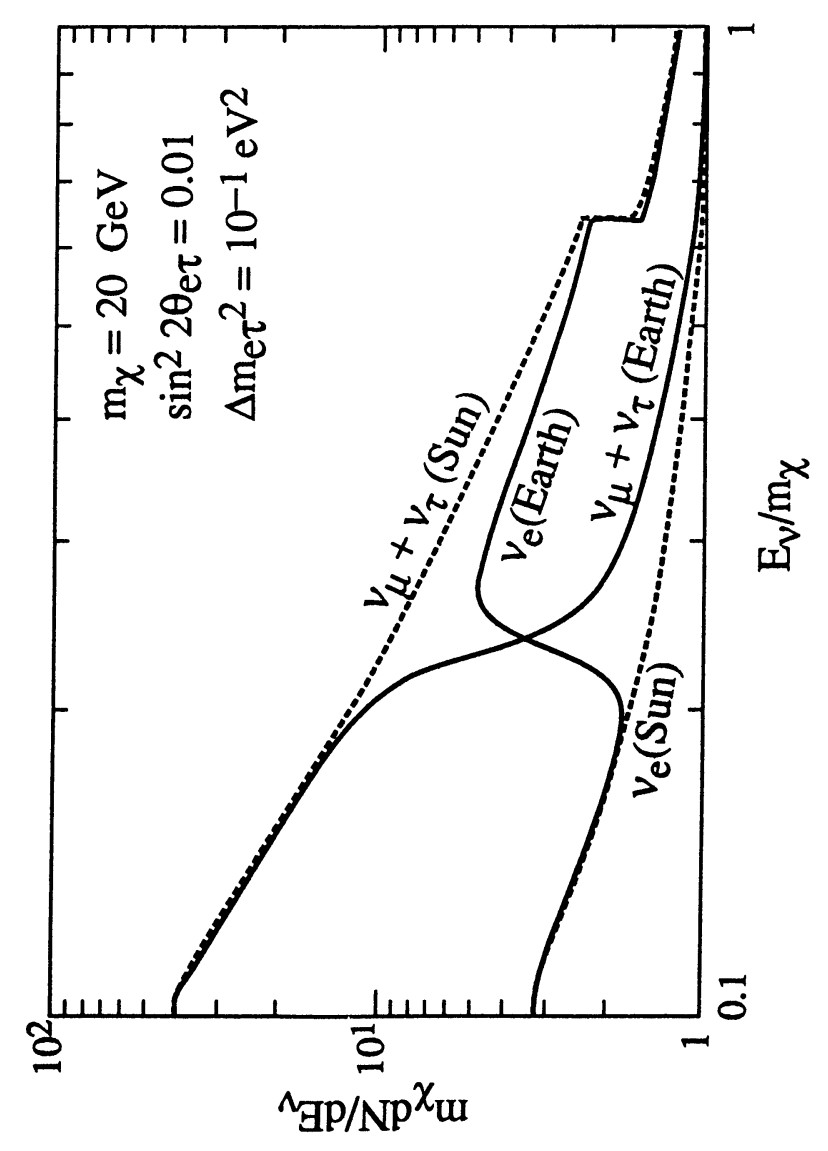

ले 